\begin{tabular}{lc} 
Journal of Environmental \\
Analysis and Progress \\
ISSN: $2525-815 \mathrm{X}$ & Journal homepage: $\underline{\text { www.jeap.ufrpe.br/ }}$ \\
\hline
\end{tabular}

\title{
Capacidade de uso da terra da sub-bacia do Córrego Maria Comprida usando Sistemas de Informações Geográficas
}

\section{Land use capacity of the sub-basin of the Maria Comprida Stream with Geographic Information System support}

\author{
Isabela do Carmo Precci Lopes ${ }^{\mathrm{a}}$, Jasmine Alves Campos ${ }^{\mathrm{a}}$ \\ ${ }^{a}$ Departamento de Engenharia Agrícola, Universidade Federal de Viçosa-UFV, Campus Universitário, Avenida PH Rolfs, \\ s/n, Viçosa, MG, Brasil. CEP: 36570-000. E-mail: isabelapreccii@gmail.com, jasminealvescampos@ gmail.com.
}

\begin{tabular}{|c|c|}
\hline A R T I C L E I N F O & A B S T R A C T \\
\hline Recebido 24 Jan 2019 & Agriculture is fundamental for the development of humanity; however, it is necessary \\
\hline Aceito 07 Fev 2019 & to adjust land use planning in order to reconcile agricultural activities with proper \\
\hline Publicado 11 Fev 2019 & soil management practices, thus reducing the impact on the environment. The study \\
\hline & aimed to evaluate the land use capacity of the Córrego Maria Comprida-MG sub- \\
\hline & Geographic Information System (GIS) was used for the framing of the lands \\
\hline & according to the system of the capacity of use. The Number of Exceeding Classes \\
\hline & use. It was verified that the sub-basin presents approximately $60 \%$ of its land in class \\
\hline & $\begin{array}{l}\text { VI of the capacity of use and that about half of the study area is being used above its } \\
\text { capacity, occupied mainly by pastures. The results proved its importance for planning }\end{array}$ \\
\hline & ne rational use of land in the Maria Comprida stream sub-basin. \\
\hline
\end{tabular}

Keywords: River basin, degradation, land use, agricultural planning.

\section{R E S U M O}

A agricultura é fundamental para o desenvolvimento da humanidade, no entanto
torna-se necessário o adequando planejamento do uso da terra de forma a conciliar
as atividades agrícolas aliadas a boas práticas de manejo do solo, possibilitando
reduzir os impactos ao ambiente. Desse modo, o estudo objetivou avaliar a
capacidade de uso da terra da sub-bacia do Córrego Maria Comprida-MG, inserida
na bacia do rio Caratinga e Rio Doce. Utilizou-se o apoio do Sistema de Informações
Geográficas (SIG) para o enquadramento das terras conforme sistema de capacide de
uso. Avaliou-se o Número de Classes Exedentes (NCE) a fim de identificar conflitos
entre a capadidade de uso e o uso atual da terra. Verificou-se que a sub-bacia
apresenta, aproximadamente, $60 \%$ das suas terras na classe VI de capacidade de uso
e que cerca da metade da área de estudo está sendo utilizada acima de sua capacidade,
ocupadas principalmente por pastagens. Os resultados mostraram-se valiosos no
planejamento do uso racional da terra da sub-bacia do córrego Maria Comprida.
Palavras-Chave: Bacia hidrográfica, degradação, uso da terra, planejamento
agrícola.

\section{Introdução}

A atividade agrícola é essencial para a vida humana por ser a base para a produção de alimentos. A agricultura evoluiu desde o início da civilização humana até os dias atuais, e está constantemente passando por mudanças, acompanhando as novas tecnologias que estão surgindo (Assad \& Almeida, 2004; Cunha \& Pinto,
2012). Portanto, o domínio dessa atividade é fundamental para que seja obtido uma boa produtividade, e além disso, não cause danos ao meio ambiente.

O conhecimento das potencialidades dos aspectos solo, do clima e do relevo é imprescindível para se determinar o adequado planejamento uso da terra de uma área, 
possibilitando mitigar o esgotamento deste recurso (Cunha \& Pinto, 2012).

$\mathrm{O}$ uso da terra em atividades que estejam em desacordo com suas potencialidades, como para fins urbano, industrial, agrícola e pecuária praticados de forma insustentáveis podem acarretar na degradação dos solos (Flauzino et al., 2016). Constituindo a erosão hídrica uma das principais causas de degradação, e relacionada a diversos impactos ao ambiente (Bertoni \& Lombardi Neto, 1999).

De acordo com Mehri et al. (2018), em áreas afetadas pelas atividades antrópicas, o planejamento do uso da terra é considerado um ato urgente para a mitigação dos impactos gerados. Neste sentido, a classificação de terras conforme a capacidade de uso é um instrumento valioso para o planejamento do uso sustentável deste recurso (Atalay, 2016).

O sistema de capacidade de uso da terra é uma classificação técnico-interpretativa, que foi originalmente desenvolvida nos Estados Unidos pela Sociedade Americana de Ciências do Solo (Klingebiel \& Montgomery, 1961). Adaptado as condições brasileiras por Lepsch et al. (1983), o sistema visa agrupar terras similares conforme suas propriedades, identificando a máxima capadidade de uso, otimizando a produção agrícola de forma eficiente e racional, e minimizando os riscos de degradação do solo (Lepsch et al., 2015).

Ademais, a interação com o Sistema de Informações Geográficas (SIG) auxilia a utilização conjunta dos fatores envolvidos na análise, tornado-se uma importante ferramenta no gerenciamento ambiental em bacias hidrográficas (Pandey et al., 2011; Santos et al., 2012). Segundo Silva et al. (2013), o SIG permite trabalhar com informações com boa acurária, reduzido custo e tempo, dando suporte ao planejamento do uso do solo, no âmbito de bacias hidrográficas.

Diante do exposto, o estudo objetivou mapear a capacidade de uso da terra da sub-bacia hidrográfica Córrego Maria Comprida, com apoio de SIG, como também verificar a conformidade entre o uso atual da terra com as classse de capacidade uso identificadas. Acredita-se que este tipo de avaliação servirá de supote aos gestores para adequarem o uso e manejo da terra nesta subbacia, possibilitado mitigar a degradação causada aos recursos naturais.

\section{Material e Métodos}

O estudo foi desenvolvido na sub-bacia do córrego Maria Comprida localizada no estado de Minas Gerais, entre as coordenadas $41^{\circ} 39^{\prime} 57^{\prime \prime} \mathrm{e}$ $41^{\circ} 40^{\prime} 55^{\prime \prime}$ de latitude sul, e $19^{\circ} 15^{\prime} 25^{\prime}$ " e $19^{\circ} 20^{\prime} 52^{\prime \prime}$ de longitude oeste, compreendendo uma área de aproximadamente $85,9 \mathrm{~km}^{2}$. A sub-bacia insere-se na porção nordeste da bacia do rio Caratinga, pertencente a bacia do Rio Doce e abrange em parte os municípios de Alvarenga e Conselheiro PenaMG (Figura 1).

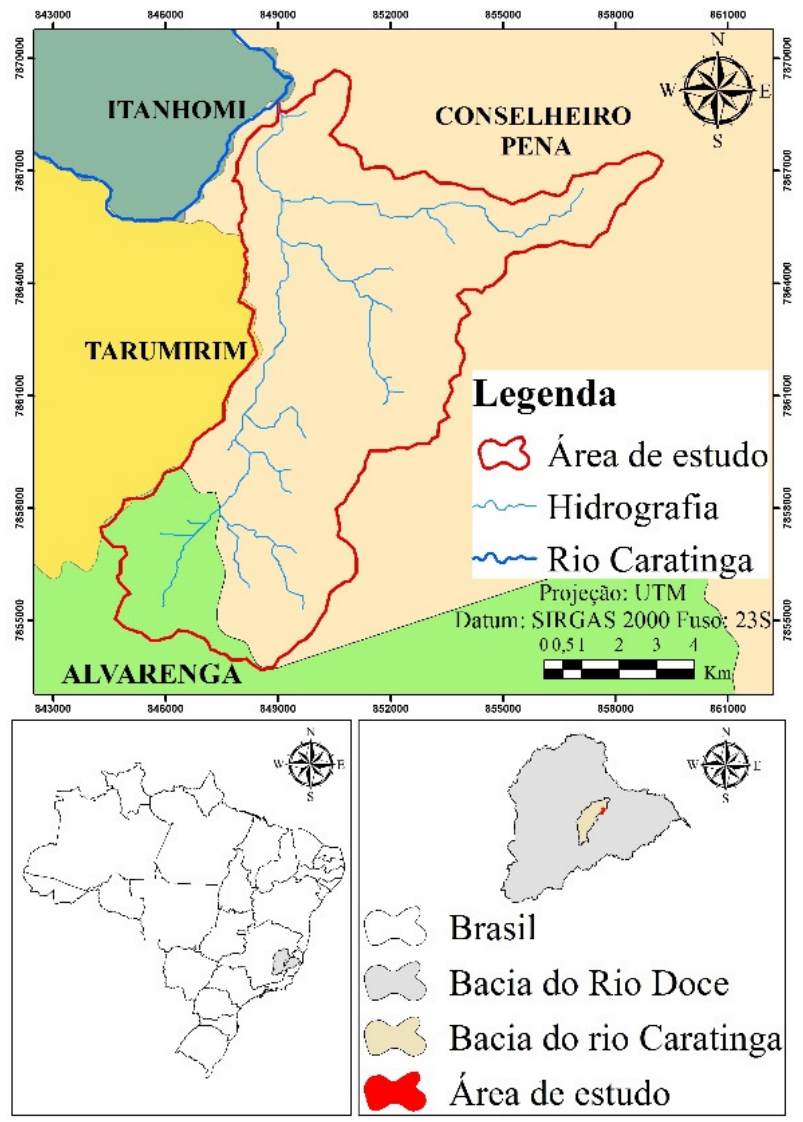

Figura 1. Mapa da localização da sub-bacia do córrego Maria Comprida, em Minas Gerais. Fonte: Lopes \& Campos (2019).

Para avaliar o sistema de capacidade de uso da terra empregou-se a metodologia proposta por Lepsch et al. (2015). Desta forma, utilizaram-se os critérios: profundidade efetiva, drenagem interna, fertilidade aparente, risco de inundação e declividade para o enquadramento das terras. A determinação das limitações de cada tipo de solo foi realizada conforme apresentado na Tabela 1.

A base de dados referente aos tipos de solo da área de estudo foi obtida do Mapa de Solo de Minas Gerais (FEAM, 2010). Foram identificados três tipos de solos na área de estudo: latossolos, neossolos e argissolos.

A declividade da sub-bacia foi gerada a partir do Modelo Digital de Elevação (MDE), do projeto Shuttle Radar Topography Mission SRTM, com resolução espacial de 30 metros, com auxílio do software ArcGIS/ArcMap ${ }^{\circledR}$ 10.1. O mapa de declividade foi classificado conforme critérios de Lepsch et al. (2015) (Tabela 1). 
Tabela 1. Enquadramento de terras em classes de capacidade de uso. Fonte: Adaptado de Lepsch et al. (2015) e Rio Grande do Sul (1979).

\begin{tabular}{|c|c|c|c|c|c|c|c|c|c|}
\hline \multirow{2}{*}{\multicolumn{2}{|c|}{ Limitações }} & \multicolumn{8}{|c|}{ Classes de capacidade de uso } \\
\hline & & $\mathbf{I}$ & II & III & IV & V & VI & VII & VIII \\
\hline \multirow{5}{*}{ Profundidade efetiva } & Muito profunda & $\mathrm{X}$ & $\mathrm{X}$ & $\mathrm{X}$ & $\mathrm{X}$ & $\mathrm{X}$ & $\mathrm{X}$ & $\mathrm{X}$ & $\mathrm{X}$ \\
\hline & Profunda & $\mathrm{X}$ & $\mathrm{X}$ & $\mathrm{X}$ & $\mathrm{X}$ & $\mathrm{X}$ & $\mathrm{X}$ & $\mathrm{X}$ & $\mathrm{X}$ \\
\hline & Moderada & & $\mathrm{X}$ & $\mathrm{X}$ & $\mathrm{X}$ & $\mathrm{X}$ & $\mathrm{X}$ & $\mathrm{X}$ & $\mathrm{X}$ \\
\hline & Rasa & & & & $\mathrm{X}$ & $\mathrm{X}$ & $\mathrm{X}$ & $X$ & $\mathrm{X}$ \\
\hline & Muito rasa & & & & & & $\mathrm{X}$ & $\mathrm{X}$ & $\mathrm{X}$ \\
\hline \multirow{5}{*}{ Drenagem interna } & Excessiva & & $\mathrm{X}$ & $\mathrm{X}$ & $\mathrm{X}$ & $\mathrm{X}$ & $\mathrm{X}$ & $\mathrm{X}$ & $\mathrm{X}$ \\
\hline & Boa & $X$ & $\mathrm{X}$ & $\mathrm{X}$ & $\mathrm{X}$ & $\mathrm{X}$ & $\mathrm{X}$ & $\mathrm{X}$ & $\mathrm{X}$ \\
\hline & Moderada & & $\mathrm{X}$ & $\mathrm{X}$ & $\mathrm{X}$ & $\mathrm{X}$ & $\mathrm{X}$ & $\mathrm{X}$ & $\mathrm{X}$ \\
\hline & Pobre & & & $\mathrm{X}$ & $\mathrm{X}$ & $\mathrm{X}$ & $\mathrm{X}$ & $\mathrm{X}$ & $\mathrm{X}$ \\
\hline & Muito pobre & & & & & $\mathrm{X}$ & $\mathrm{X}$ & $X$ & $\mathrm{X}$ \\
\hline \multirow{5}{*}{ Fertilidade aparente } & Muito alta & $\mathrm{X}$ & $\mathrm{X}$ & $\mathrm{X}$ & $\mathrm{X}$ & $X$ & $\mathrm{X}$ & $\mathrm{X}$ & $\mathrm{X}$ \\
\hline & Alta & $X$ & $\mathrm{X}$ & $\mathrm{X}$ & $\mathrm{X}$ & $\mathrm{X}$ & $\mathrm{X}$ & $\mathrm{X}$ & $\mathrm{X}$ \\
\hline & Média & & $\mathrm{X}$ & $\mathrm{X}$ & $\mathrm{X}$ & $\mathrm{X}$ & $\mathrm{X}$ & $\mathrm{X}$ & $\mathrm{X}$ \\
\hline & Baixa & & & $\mathrm{X}$ & $\mathrm{X}$ & & $\mathrm{X}$ & $\mathrm{X}$ & $\mathrm{X}$ \\
\hline & Muito baixa & & & & & & $\mathrm{X}$ & $X$ & $\mathrm{X}$ \\
\hline \multirow{3}{*}{ Risco de inundação } & Ocasional & & & $\mathrm{X}$ & & $\mathrm{X}$ & & & $\mathrm{X}$ \\
\hline & Frequente & & & & & $\mathrm{X}$ & & & $X$ \\
\hline & Muito frequente & & & & & & & & $\mathrm{X}$ \\
\hline \multirow{7}{*}{ Declividade (\%) } & $0-2$ & $\mathrm{X}$ & $\mathrm{X}$ & $\mathrm{X}$ & $\mathrm{X}$ & $\mathrm{X}$ & $\mathrm{X}$ & $\mathrm{X}$ & $\mathrm{X}$ \\
\hline & $2-5$ & & $\mathrm{X}$ & $\mathrm{X}$ & $\mathrm{X}$ & & $\mathrm{X}$ & $\mathrm{X}$ & $\mathrm{X}$ \\
\hline & $5-10$ & & & $\mathrm{X}$ & $\mathrm{X}$ & & $\mathrm{X}$ & $\mathrm{X}$ & $\mathrm{X}$ \\
\hline & $10-15$ & & & & $\mathrm{X}$ & & $\mathrm{X}$ & $\mathrm{X}$ & $\mathrm{X}$ \\
\hline & $15-45$ & & & & & & $\mathrm{X}$ & $\mathrm{X}$ & $X$ \\
\hline & $45-70$ & & & & & & & $\mathrm{X}$ & $\mathrm{X}$ \\
\hline & Superior a 70 & & & & & & & & $\mathrm{X}$ \\
\hline
\end{tabular}

Obtidos os mapas de capacidade para os fatores do solo e declividade, essas informações foram cruzadas no raster calculador do ArcGIS/ArcMap ${ }^{\circledR}$, considerando o fator mais limitante, ou seja, em cada pixel utilizou-se o maior valor obtido na classificação dos fatores solo e declividade (Campos et al., 2018). Este procedimento permitiu determinar a capacidade do uso e manejo da terra do córrego Maria Comprida. Imagens Landsat 8 OLI (Operational Land Imager) com resolução de $30 \mathrm{~m}$ foram utilizadas para realizar a classificação supervisionada por máxima verossimilhança do uso e cobertura das terras da sub-bacia. Nesta classificação, realizou-se a distinção em sete classes: hidrografia, reflorestamento, vegetação nativa, solo exposto, área urbana, área agrícola e pastagem. A acurácia da classificação foi verificada pelo índice Kappa (Landis \& Koch, 1977).

Visando a determinação das classes do uso atual da terra, retirou-se da análise os usos referentes a área urbana e a hidrografia, uma vez que estes não estão atrelados à utilização agrícola. Foram associadas as classes que melhor representavam suas condições aos demais tipos de uso e cobertura.

Em seguida, realizou-se, com auxílio da ferramenta raster calculador do software ArcGIS/ArcMap ${ }^{\circledR}$, a subtração entre o mapa de capacidade de uso e o de uso atual da terra, resultando no mapa do Número de Classes Excedentes (NCE). Deste modo, as áreas que apresentavam NCE com valores negativos indicavam terras subutilizadas, aquelas com valores positivos constituíam terras sobreutilizadas e aquelas com valores nulos seriam terras utilizadas, conforme a sua capacidade de uso e manejo (Campos et al., 2018; Monteiro, 2016).

\section{Resultados}

O mapeamento da capacidade das terras da sub-bacia do córrego Maria Comprida quanto ao fator declividade é apresentado na Figura 2. Verifica-se o predomínio das menores classes de declividades, principalmente próximas aos cursos dos rios, enquanto as maiores encontram-se distribuídas em toda a área de estudo. 


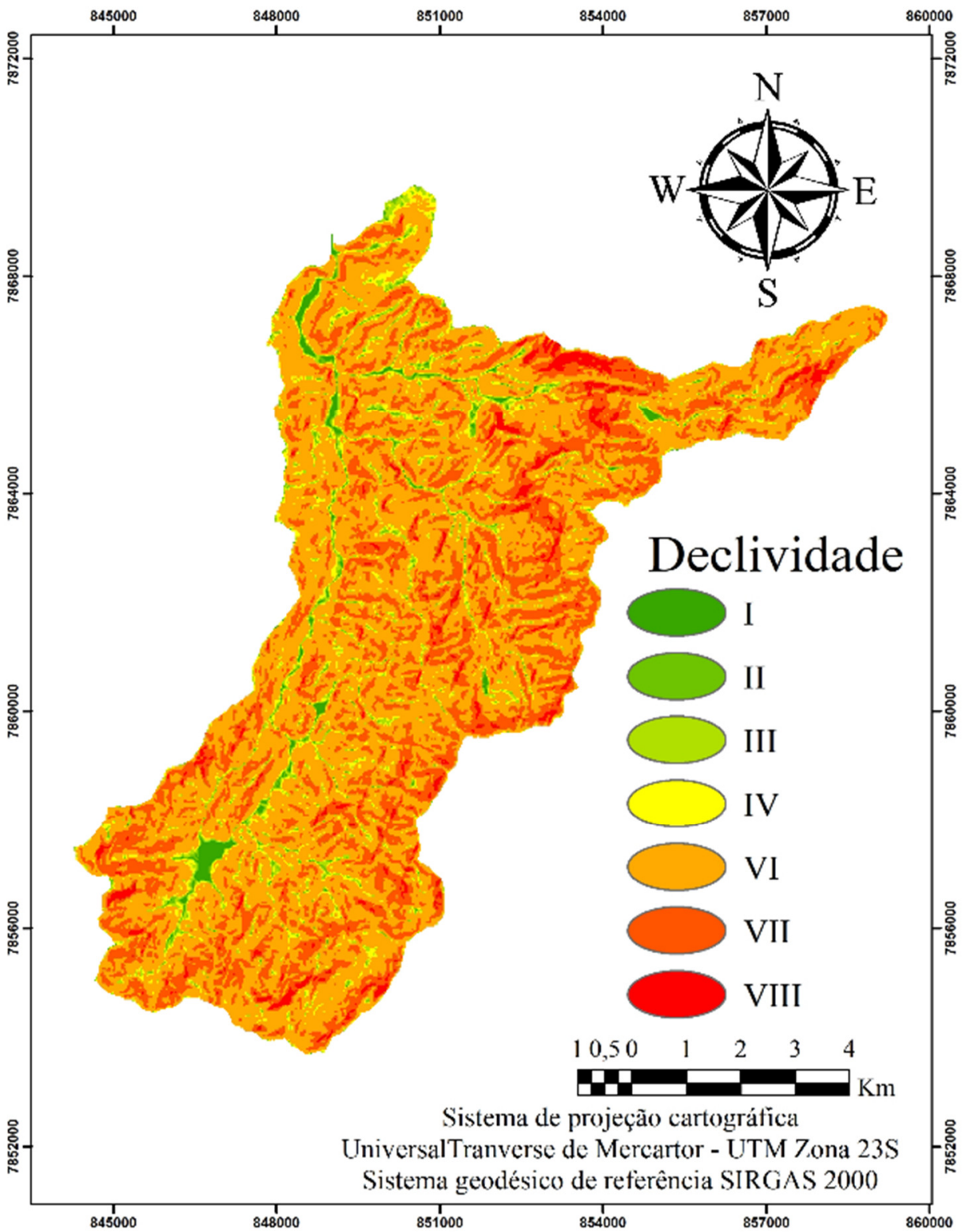

Figura 2. Classes de capacidade de uso, quanto ao fator declividade, na sub-bacia do córrego Maria Comprida, em Minas Gerais. Fonte: Lopes \& Campos (2019).

Na Tabela 2 estão dispostos os percentuais de área referente a cada classe de declividade. Observa-se que, aproximadamente, $60 \%$ das terras foram classificadas como classe VI para este fator, por possuírem uma declividade de 15 a $45 \%$.

Tabela 2. Área da sub-bacia do córrego Maria Comprida, em Minas Gerais, em cada classe de declividade.

\begin{tabular}{ccc}
\hline $\begin{array}{c}\text { Declividade } \\
(\boldsymbol{\%})\end{array}$ & Classes & $\begin{array}{c}\text { Área da sub-bacia } \\
\left(\mathbf{k m}^{2}\right)\end{array}$ \\
\hline $0-2$ & I & 0,94 \\
$2-5$ & II & 1,14 \\
$5-10$ & III & 2,62 \\
$10-15$ & IV & 3,9 \\
$15-45$ & VI & 51,41 \\
$45-70$ & VII & 23,59
\end{tabular}

$\begin{array}{ll}>70 \quad \text { VIII } & 2,3 \\ \text { Considerando o solo, identificaram-se três }\end{array}$ tipos na região da sub-bacia. Os argissolos vermelho amarelo eutrofico (PVAe) corresponde ao maior percentual, representando $81,6 \%$ da área, seguido pelos neossolos litólicos com 13,85\% da área e, em menor quantidade, os latossolos vermelho amarelo distróficos, correspondendo a $4,54 \%$.

Realizando o enquadramento das terras conforme apresentado na Tabela 1, pode-se determinar para cada tipo de solo a classe mais limitante conforme os critérios avaliados, como é mostrado na Tabela 3. A distribuição das classes de capacidade de uso quanto ao fator solo para a subbacia do córrego Maria Comprida pode ser visualizado na Figura 3. 


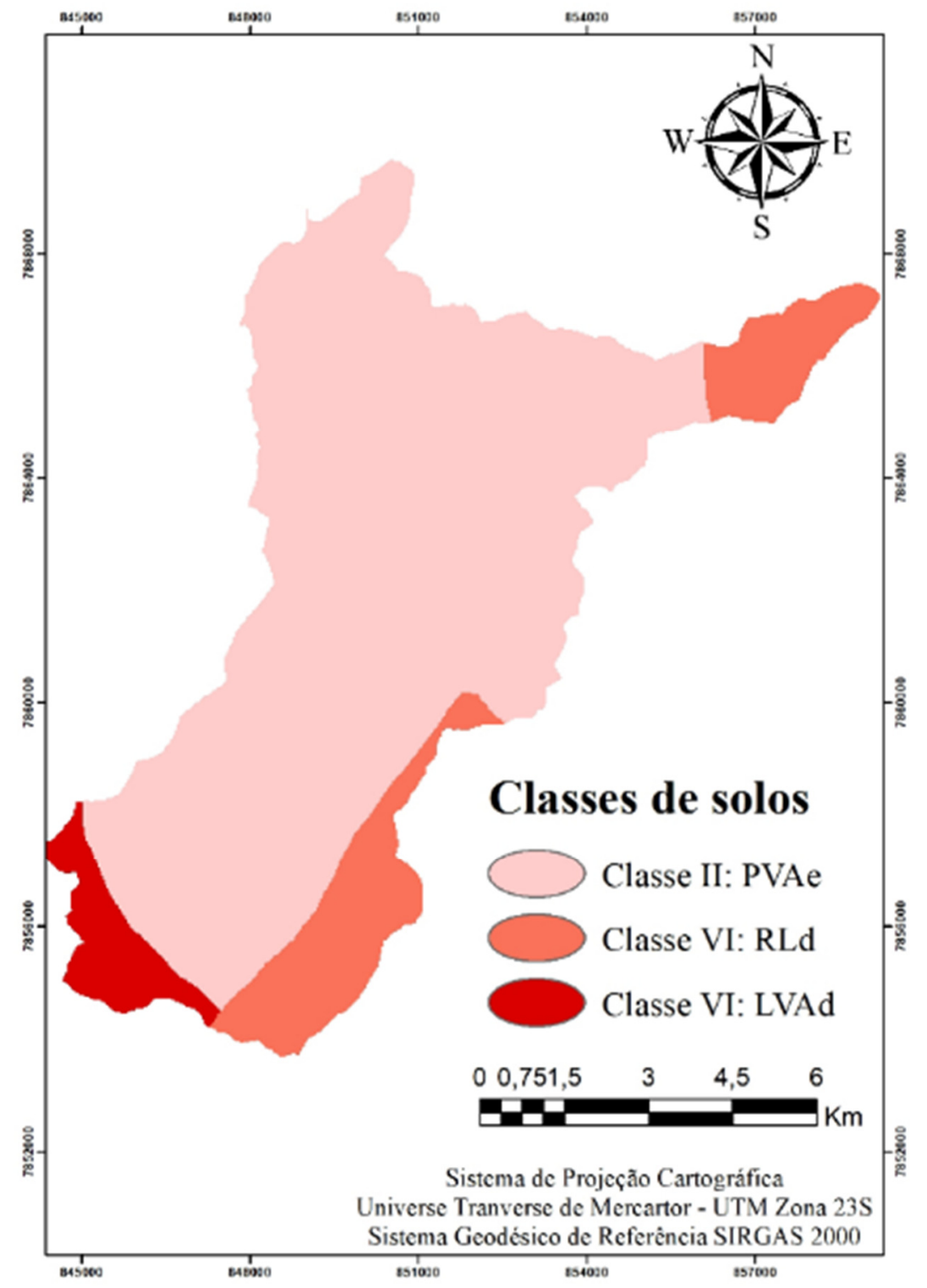

Figura 3. Classes de capacidade de uso, quanto ao fator solo, na sub-bacia do córrego Maria Comprida, em Minas Gerais. Fonte: Lopes \& Campos (2019).

Tabela 3. Enquadramento dos tipos de solos, quanto à capacidade de uso, na sub-bacia do córrego Maria Comprida, em Minas Gerais. Fonte: Lopes \& Campos (2019).

\begin{tabular}{lcccccc}
\hline Tipo de solo & $\begin{array}{c}\text { Profundidade } \\
\text { efetiva }\end{array}$ & $\begin{array}{c}\text { Drenagem } \\
\text { interna }\end{array}$ & $\begin{array}{c}\text { Risco de } \\
\text { inundação }\end{array}$ & $\begin{array}{c}\text { Fertilidade } \\
\text { aparente }\end{array}$ & $\begin{array}{c}\text { Classe } \\
\text { limitante }\end{array}$ & $\%$ \\
\hline Latossolo vermelho amarelo & I & I & - & VI & VI & 4,54 \\
distrófico & VI & II & - & II & VI & 13,86 \\
Neossolo litólico distrófico & II & II & - & I & II & 81,60 \\
Argissolo vermelho amarelo & & & & &
\end{tabular}

Desta forma, após o cruzamento dos mapas de capacidade quanto aos fatores declividade e de solos foi possível gerar o mapeamento da capacidade de uso e manejo das terras da sub-bacia do córrego Maria Comprida, apresentado na Figura 4. Os valores correspondentes à área da sub-bacia e seu respectivo percentual referente a cada classe de capacidade de uso da terra são representados na Tabela 4.
Tabela 4. Área da sub-bacia correspondente a cada classe de capacidade de uso.

\begin{tabular}{cc}
\hline Classes & $\mathbf{k m}^{\mathbf{2}}$ \\
\hline II & 2,0 \\
III & 2,4 \\
IV & 3,4 \\
VI & 52,2 \\
VII & 23,6 \\
VIII & 2,3 \\
\hline
\end{tabular}




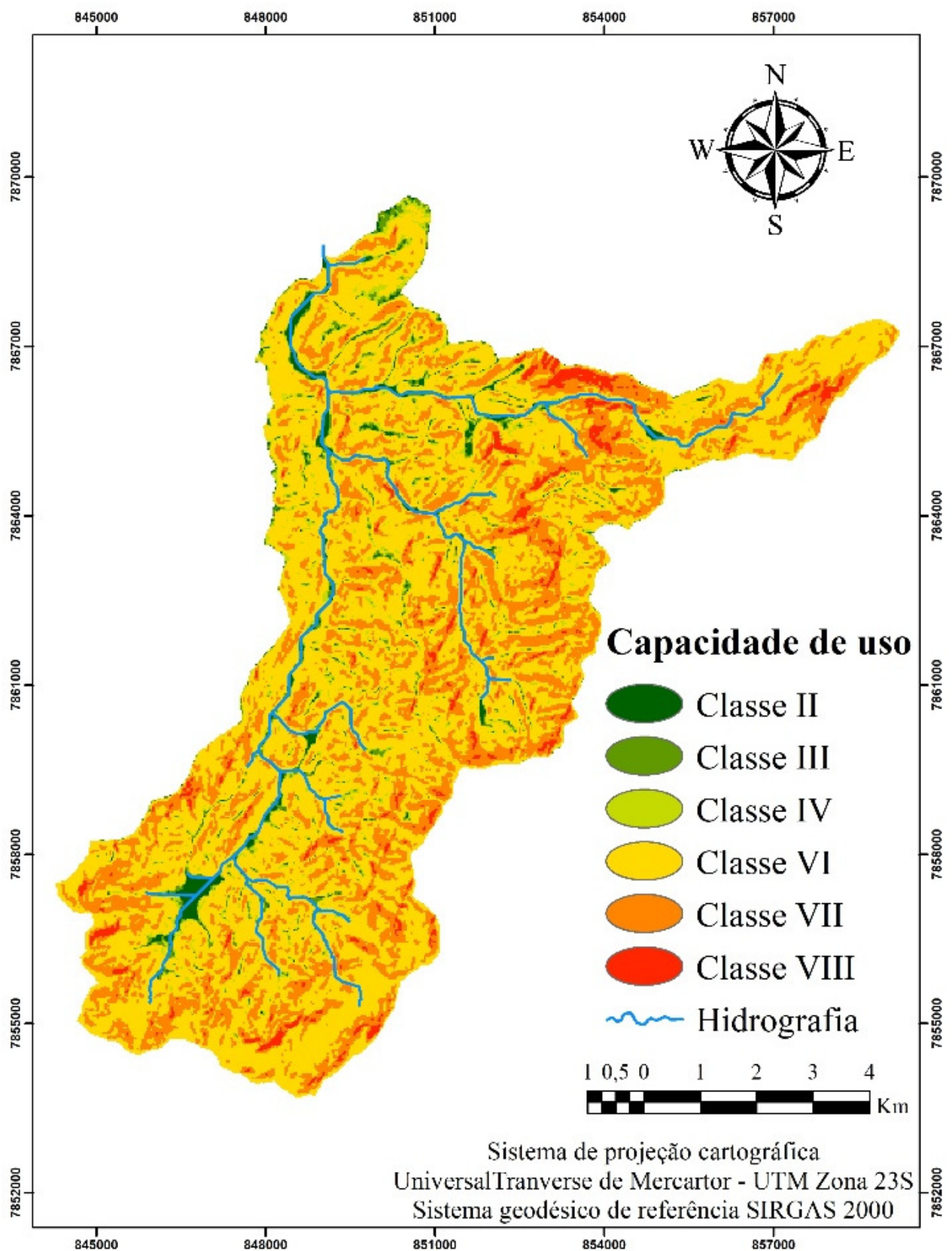

Figura 4. Classes de capacidade de uso das terras na sub-bacia do córrego Maria Comprida, em Minas Gerais. Fonte: Lopes \& Campos (2019).

$\mathrm{Na}$ Tabela 4 verificamos que a classe predominante de capacidade de uso e manejo da terra para a sub-bacia do córrego Maria Comprida é a classe VI, correspondendo a aproximadamente $60,8 \%$ da área. Esse fato está associado a maior parte da declividade da sub-bacia se encontrar na classe VI, com valores de 15 a $45 \%$ de declividade. Além disso, dois dos três tipos de solos da bacia possuem a classe VI como limitante.

Avaliando a acurácia da imagem do uso e cobertura da terra pelo índice Kappa, obteve-se o valor de 0,89 , o que indica excelente representatividade da classificação da imagem com o uso real da terra.
$\mathrm{Na}$ classificação do uso atual da terra, associou-se a cada tipo de uso as classes que representavam melhor as condições da bacia. Sendo assim, determinou-se a classe I para representar solo exposto; classe V para pastagem que, em geral, apresentavam degradadas; classe VI para agricultura, constituídas, principalmente, de culturas permanentes; classe VII para a floresta plantada; e a classe VIII para vegetação nativa. $\mathrm{Na}$ Figura 5 é apresentado o mapa de uso atual da terra e suas correspondentes classes conforme a sistema de capacidade de uso. 


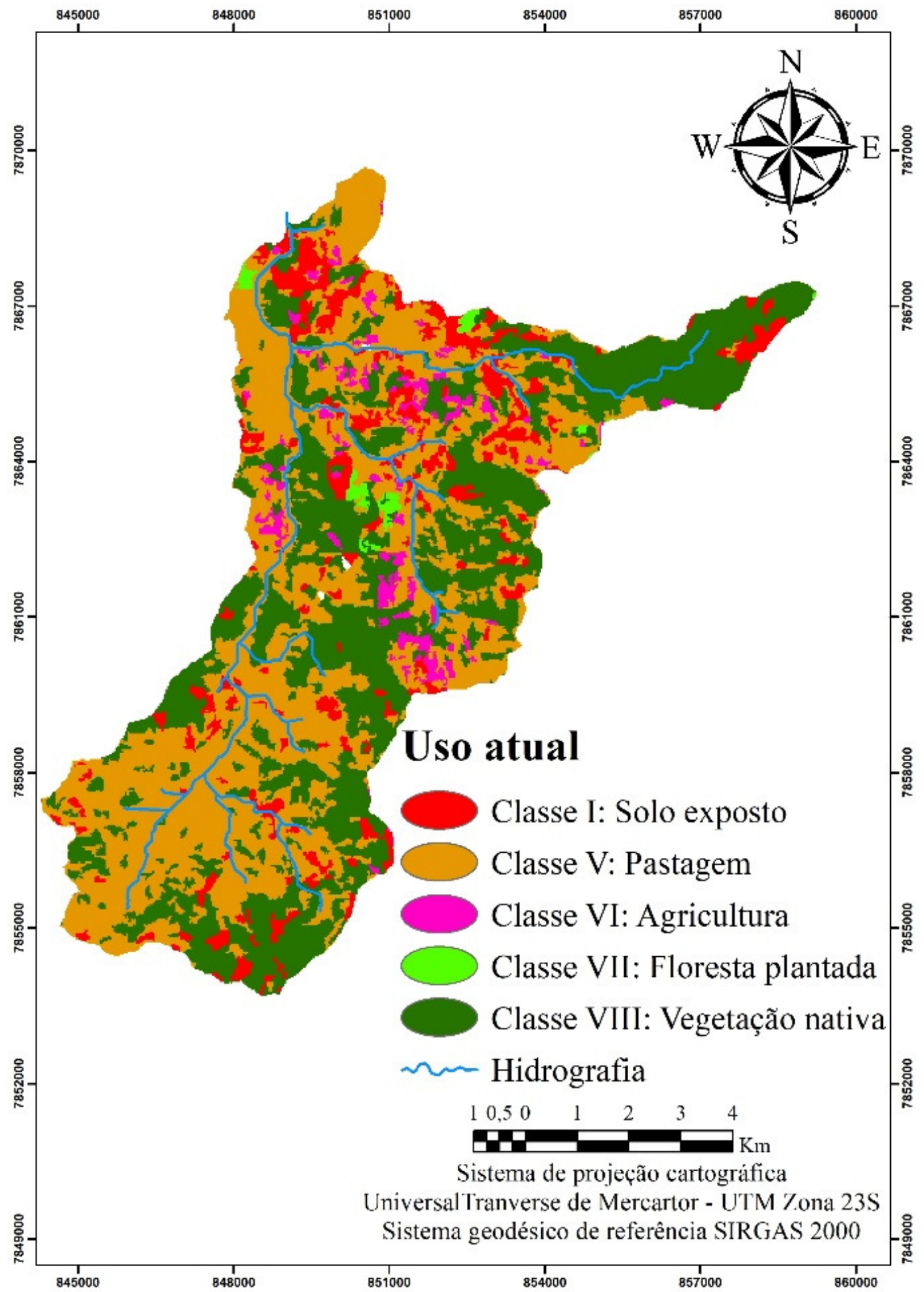

Figura 5. Classes do uso atual da terra na sub-bacia do córrego Maria Comprida, em Minas Gerais. Fonte: Lopes \& Campos (2019).

O mapeamento obtido na avaliação do Número de Classes Excedentes (NCE) é apresentado na Figura 6, que indica o nível de exploração das terras. Verifica-se que os valores de NCE na sub-bacia em estudo variaram de -1 a 7. 


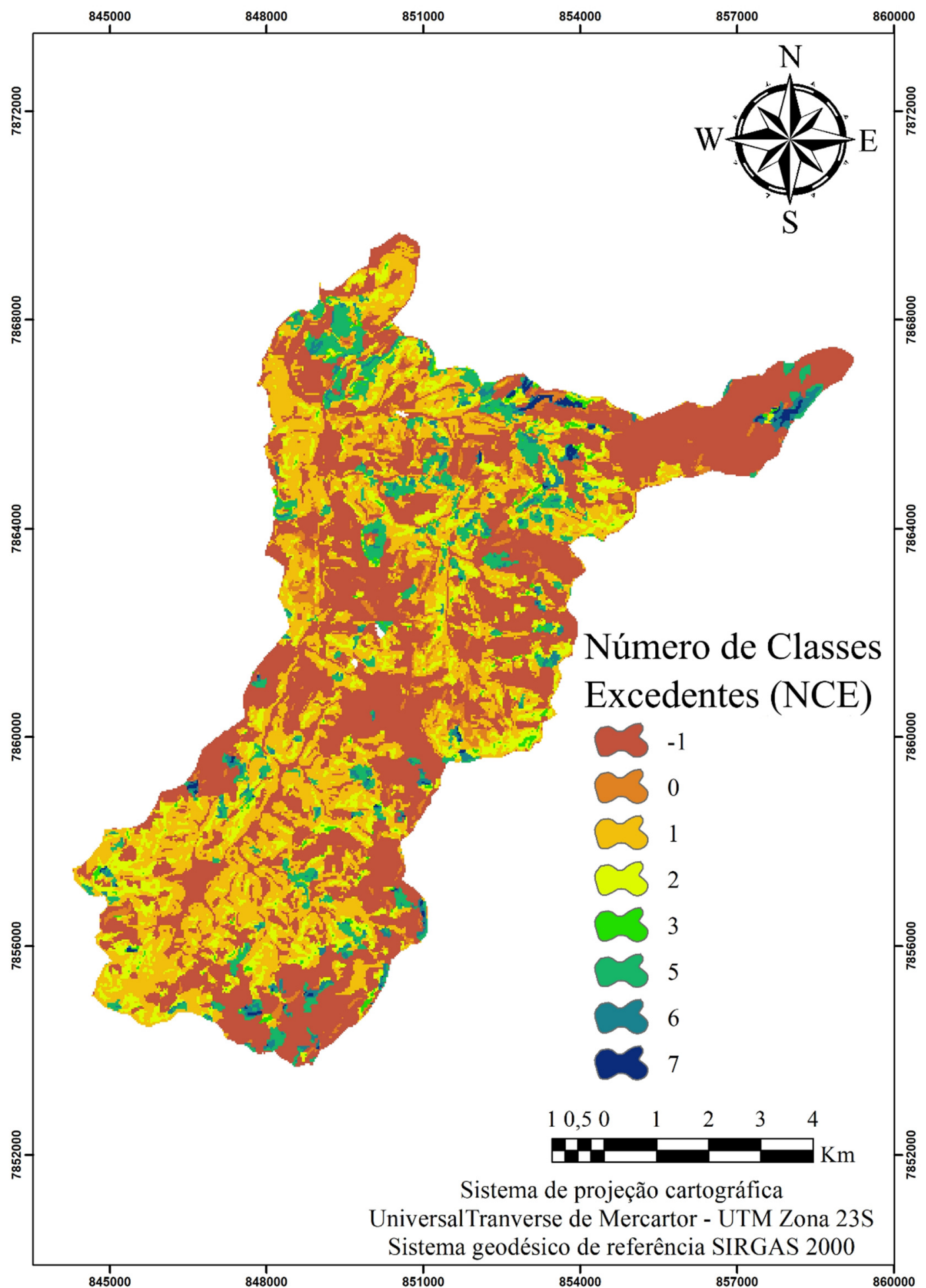

Figura 6. Número de classes excedentes (NCE) na sub-bacia do córrego Maria Comprida, em Minas Gerais. Fonte: Lopes \& Campos (2019).

Para facilitar a compreensão, levou-se em consideração que todos os números positivos são representados por 1 , o que indica a utilização atual de grande das terras acima da sua capacidade de uso e manejo. Assim, na Tabela 5 estão apresentadas as três classes de NCE e suas respectivas áreas.
Tabela 5. Área da sub-bacia do córrego Maria Comprida, em Minas Gerais, conforme valores de NCE.

\begin{tabular}{cc}
\hline $\mathbf{N C E}$ & $\mathbf{k m}^{\mathbf{2}}$ \\
\hline-1 & 39,15 \\
0 & 2,72 \\
1 & 43,97 \\
\hline
\end{tabular}


Analisando a Tabela 5, verifica-se que $45,61 \%$ da área agricultável da sub-bacia $(39,15$ $\mathrm{km}^{2}$ ) apresenta valor de NCE negativo (-1), indicando a subutilização da área, ou seja, a existência de exploração da terra abaixo da capacidade de uso. Estas áreas são representadas por locais que apresentam uso menos intensivos, como áreas de vegetação nativa, mas que poderiam ser utilizadas para fins agrícolas, em exceção das áreas protegidas por lei ambientais.

Em $3,16 \%$ da área agricultável, representada por $2,72 \mathrm{~km}^{2}$, foi obtido valor nulo para o NCE, representando uma área que, atualmente, está no limite da capacidade do uso e manejo da terra. Considerando a maior parte da sub-bacia, 51,23\% da área agricultável, ou seja, $43,97 \mathrm{~km}^{2}$, apresentaram valor positivo para NCE. Assim, essas terras são classificadas como áreas intensamente exploradas, utilizadas acima da sua capacidade de suporte e necessitam que sejam implementadas práticas conservacionistas para sua restauração.

A Tabela 6 apresenta a intensidade de utilização das terras, de acordo com cada uso e ocupação da sub-bacia estudada. Verifica-se que nas áreas com subutilização, o principal uso da terra refere-se à vegetação nativa, representando $80,65 \%$.

Tabela 6. Percentual de área de utilização do solo, em cada classe de uso atual da terra, na sub-bacia do Córrego Maria Comprida, em Minas Gerais.

\begin{tabular}{lccc}
\hline $\begin{array}{l}\text { Uso e ocupação da } \\
\text { terra }\end{array}$ & $\begin{array}{c}\text { Áreas com } \\
\text { subutilização }\end{array}$ & $\begin{array}{c}\text { Áreas de utilização conforme } \\
\text { capacidade de uso } \\
(\%)\end{array}$ & $\begin{array}{c}\text { Áreas com } \\
\text { sobreutilização }\end{array}$ \\
\hline Reflorestamento & 1,07 & 6,15 & 0,17 \\
Vegetação nativa & 80,65 & 39,37 & 2,96 \\
Solo exposto & 0,68 & 1,31 & 18,81 \\
Agricultura & 0,98 & 48,21 & 2,53 \\
Pastagem & 16,62 & 4,97 & 75,53 \\
\hline
\end{tabular}

Nestes locais, nos quais os usos da terra estão de acordo com a capacidade de uso, predominam a agricultura, correspondendo a 48,21\%. O principal uso das áreas com sobreutilização refere-se à pastagem, correspondendo à 75,53\%. Assim, este resultado permite avaliar os usos que necessitam de atenção prioritária na adequação e planejamento agrícola.

\section{Discussão}

A classificação das terras de acordo com o sistema de capacidade de uso e manejo foi realizada com base nos dados de declividade e tipos de solo encontrado na área de estudo. Assim, analisando o fator declividade, verificamos que o relevo da sub-bacia do córrego Maria Comprida, em sua maior parte, é classificado por Lepsch et al. (2015) como fortemente inclinado.

Aires et al. (2017) também ressaltaram a relevância da declividade no estudo da capacidade de uso das terras de uma microbacia do rio Piracicaba, em Minas Gerais. Os autores relataram o fator declividade como o aspecto mais limitante na classificação das terras, em que cerca de $77 \%$ da área de estudo apresentava relevo fortemente ondulado e montanhoso (Aires et al., 2017).

De acordo com Mendonça (1999), o conhecimento das inclinações do relevo de uma área é fundamental para o estudo do uso e cobertura da terra. Além disso, permiti compreender a ocorrência dos processos erosivos no solo, como também o transporte dos sedimentos (Mendonça, 1999).

Quanto ao fator solo, os argissolos vermelho amarelos eutróficos (PVAe) estão presentes no maior percentual da área de estudo, ocupando $81,60 \%$. Apesar de serem caracterizados pela presença do horizonte B textural, com acúmulo de argila (EMBRAPA, 2013), foram enquadrados na classe limitante II. Os PVAe, por serem típicos de relevo forte ondulado, possibilita a moderada drenagem do solo e por serem eutróficos a fertilidade aparente foi considerada alta.

Os neossolos litólicos (RLd) correspondem a 13,86\% da área, são característicos por serem solos jovens e, portanto, considerados rasos (Sartoni et al., 2005). Desta forma, a profundidade constitui a característica mais limitante, classificando-os como na classe VI.

Em menor percentual estão os latossolos vermelho amarelos distróficos (LVAd), correspondendo a 4,54\% da sub-bacia. Em relação a profundidade, este tipo de solo é caracterizado por serem profundos ou muito profundos, no entanto por apresentarem alto grau de intemperismo, possuem baixa fertilidade (EMBRAPA, 2013). Analisando essas características, este tipo de solo também foi enquadrado na classe limitante VI. 
Sendo assim, esses fatores permitiram o enquadramento do maior percentual da área da subbacia na classe de capacidade de uso VI. Essa classe constitui terras com limitações severas, podendo ser usadas apenas para pastagens, florestas cultivadas/nativas, ou ainda em determinadas culturas permanentes (Lepsch et al., 2015; Rio Grande do Sul, 1979). Portanto, são áreas que necessitam da adoção de medidas de conservação do solo e água, como também requer um adequado planejamento do uso da terra.

Resultado semelhante também foi obtido para a sub-bacia hidrográfica do ribeirão José Pereira, em Minas Gerais, por Flauzino et al. (2016). Esses autores verificaram grande percentual da área de estudo, $71 \%$, pertence à classe VI, conforme sistema de capacidade de uso e manejo da terra (Flauzino et al., 2016).

Na sub-bacia hidrográfica do rio Pomba, inserida nos estados de Minas gerais e Rio de Janeiro, Almeida (2014) também constatou a predominância da classe VI de capacidade de uso da terra, $41,36 \%$, relacionando a este fato existe a ocorrência de relevo acentuado na área de estudo.

Analisando a existência de conflitos entre a capacidade de uso e o uso atual da terra, verificamos uma expressiva área da sub-bacia do córrego Maria Comprida utilizada em não conformidade com a classificação. Aires et al. (2017) também obtiveram resultado similar, no qual verificaram que $68,6 \%$ da área de estudo apresentava inconformidade com a classificação de terras, sendo utilizadas principalmente como pastagens.

Práticas degradantes, como o sobrepastejo, a ausência da correção da fertilidade do solo e o relevo acidentado são relatadas como principais desencadeadores dos processos erosivos nas áreas de passagem da região do rio Doce (Ferreira, 2016; Silva et al., 2010). Sendo assim, o emprego da metodologia utilizada no presente estudo mostrouse importante na análise das reais condições da subbacia, demonstrando a necessidade da adequação entre o uso da terra e a capacidade de uso, especialmente em áreas destinadas à pastagem.

Desta forma, diante dos resultados obtidos os gestores, atuando em nível de gerenciamento de bacias hidrográficas, é possível propor estratégias e destinar recursos para a realização de programas destinados a adequar e restaurar o uso das áreas de pastagens do córrego Maria Comprida. A adoção de práticas conservacionistas de caráter edáfico, vegetativo e mecânico devem ser consideradas, uma vez que estas constituem importantes ferramentas na manutenção e melhoria da fertilidade, proteção e minimização das perdas de solo e redução da velocidade de escoamento superficial da água (Pruski et al., 2009).

Além disso, a integração dos produtores agrícolas aos programas de conservação dos recursos naturais, como os Pagamentos por Serviços Ambientais, pode ser importante para o sucesso dessas ações (Manfré et al., 2013).

\section{Conclusão}

$\mathrm{O}$ uso do SIG se mostrou eficiente no mapeamento e avaliação da capacidade de uso e manejo das terras da sub-bacia do córrego Maria Comprida, sendo possível verificar que, aproximadamente, $60 \%$ das terras se enquadram na classe VI.

Aproximadamente metade da área agricultável da sub-bacia está sendo sobreutilizada, ou seja, existem grandes áreas que estão sendo demasiadamente exploradas e que apresentam inconformidade entre a capacidade e o uso da terra. Isto pode dar ênfase às pastagens, que constituem o uso predominantes nestas áreas.

$\mathrm{O}$ estudo mostrou-se valioso no planejamento do uso racional da terra da sub-bacia do córrego Maria Comprida. Um adequado plano de manejo de pastagens deve ser empregado por parte dos órgãos gestores da sub-bacia, o que possibilitará melhorar a produtividade e reduzir a degradação do solo.

\section{Agradecimentos}

Os autores agradecem o apoio da Coordenação de Aperfeiçoamento de Pessoal de Nível Superior - Brasil (CAPES), Código de Financiamento 001, e da Fundação de Amparo à Pesquisa do Estado de Minas Gerais (FAPEMIG).

\section{Referências}

AIRES, U. R. V.; REZENDE, C. H. da S.; SILVA, J. L. de A.; CAMPOS, J. A.; SANTOS, C. A. dos. 2017. Capacidade do uso da terra: um estudo de caso em uma microbacia do Rio Piracicaba, MG. Nativa, v. 5, n. 6, p. 402-409.

ALMEIDA, R. 2014. A. Índice para caracterizar a adequação da ocupação do solo à sua capacidade de uso. Dissertação de Mestrado, Universidade Federal de Viçosa. Viçosa, Minas Gerais, Brasil. 46p.

ASSAD, M. L. L.; ALMEIDA, J. 2004. Agricultura e Sustentabilidade: contexto, desafios e cenários. Ciência \& Ambiente, n. 29, p. 15-30.

ATALAY, I. 2016. A New Approach to the Land Capability Classification: Case Study of Turkey. Procedia Environmental Science, v. 32, p. 264-274. 
BERTONI, J. C.; LOMBARDI NETO, F. 1990. Conservação do solo. São Paulo: Ícone, 355p.

CAMPOS, J. A. 2018. Fragilidade Ambiental e Capacidade de Uso da Terra da Bacia Hidrográfica do Rio Caratinga, MG. Dissertação de Mestrado, Universidade Federal de Viçosa. Viçosa, Minas Gerais, Brasil. 83p.

CUNHA, C. M. L.; PINTON, L. G. 2012. Avaliação da capacidade de uso da terra da bacia do Córrego do Cavalheiro-Analândia, SP. Geociências, v. 31, n. 3, p. 459-471.

EMBRAPA - Empresa Brasileira de Pesquisa Agropecuária. 2013. Sistema Brasileiro de Classificação de Solos. 3. ed. Brasília: EMBRAPA, Brasília, 353p.

FEAM - Fundação Estadual do Meio Ambiente. 2010. Mapa de solos do Estado de Minas Gerais. Belo Horizonte: Fundação Estadual do Meio Ambiente, UFV/CETEC/UFLA/FEAM. 49p.

FERREIRA, T. 2016. Boas práticas para a agricultura familiar na Bacia do Rio Doce. Rio de Janeiro: Instituto Bioatlântica, 42p.

FLAUZINO, B. K.; MELLONI, E. G. P.; PONS, N. A. D.; LIMA, O. de. 2016. Mapeamento da capacidade de uso da terra como contribuição ao planejamento de uso do solo em sub-bacia hidrográfica piloto no sul de Minas Gerais. Geociências, v. 35, n. 2, p. 277-287.

KLINGEBIEL, A. A.; MONTGOMERY, P. H. 1961. Land-capability Classification. Agricultural Handbook, n. 210, Soil Conservation Service. Washington, DC. 21p.

LANDIS, J. R.; KOCH, G. G. 1977. The measurement of observe agreement for categorical data. Biometrics, v. 31, p. 159-174.

LEPSCH, I. F.; BELLINAZZI JR., R.; BERTOLINI, D.; ESPÍNDOLA, C. R. 1983. Manual para levantamento utilitário do meio físico e classificação de terras no sistema de capacidade de uso. Campinas: SBCS. 175p.

LEPSCH, I. F.; ESPINDOLA, C. R.; VISCHI FILHO, O. J.; HERNANI, L. C.; SIQUEIRA, D. S. 2015. Manual para levantamento utilitário e classificação de terras no sistema de capacidade de uso. Viçosa, MG: SBCS. 170p.
MANFRÉ, L. A.; SILVA, A. M. da; URBAN, R. C.; RODGERS, J. 2013. Environmental fragility evaluation and guidelines for environmental zoning: a study case on Ibiuna (the Southeastern Brazilian region). Environmental Earth Sciences, v. 69, n. 3, p. 947-957.

MEHRI, A.; SALMANMAHINY, A.; TABRIZI, A. R. M.; MIRKARIMI, S. H.; SADODDIN, A. 2018. Investigation of likely effects of land use planning on reduction of soil erosion rate in river basins: Case study of the Gharesoo River Basin. CATENA, v. 167, p. 116-129.

MENDONÇA, F. 1999. Diagnóstico e análise ambiental de microbacia hidrográfica: Proposição metodológica na perspectiva do zoneamento, planejamento e gestão ambiental. Rev. Raega. v. 3, n. 3, p. 67-89.

MONTEIRO, L. I. B. 2016 Pagamento por serviços ambientais em condições de uso intensivo do solo. Dissertação de Mestrado, Universidade Federal de Viçosa, Viçosa, Minas Gerais, Brasil. 70p.

PANDEY, A.; CHOWDARY, V. M.; MAL, B. C.; DABRAL, P. P. 2011. Remote sensing and gis for identification of suitable sites for soil and water conservation structures. Land Degradation \& Development, v. 22, n. 3, p. 359-372.

PRUSKI, F. F. 2009. Conservação de solo e água: práticas mecânicas para o controle da erosão hídrica. Viçosa: UFV. 279p.

RIO GRANDE DO SUL. 1979. Manual de conservação do solo. Porto Alegre: Secretaria da Agricultura, pp. 42-72.

SANTOS, P. G.; BERTOL, I.; CAMPOS, M. L.; RAFAELI NETO, S. L.; MAFRA, A. L. 2012. Classificação de terras segundo sua capacidade de uso e identificação de conflitode uso do solo em microbacia hidrográfica. Revista de Ciências Agroveterinárias, v. 11, n. 2, p. 146-157.

SARTONI, A.; LOMBARDI, F.; GENOVEZ, A. M. 2005. Classificação Hidrológica de Solos Brasileiros para a Estimativa da Chuva Excedente com o Método do Serviço de Conservação do Solo dos Estados Unidos Parte 1: Classificação. Revista Brasileira de Recursos Hídricos, v. 10, n. 4, p. 0518.

SILVA, M. A.; FREITAS, D. A. F. de; SILVA, M. L. N.; OLIVEIRA, A. H.; LIMA, G. C.; CURI, N. 2013. Sistema de informações geográficas no 
planejamento de uso do solo. Revista Brasileira de Ciências Agrárias, v. 8, n. 2, , p. 316-323.

SILVA, M. A. da; SILVA, M. L. N.; CURI, N.; SANTOS, G. R. dos; MARQUES, J. J. G. de S. e
M.; MENEZES, M. D. de; LEITE, F. P. 2010. Avaliação e espacialização da erosividade da chuva no Vale do Rio Doce, região centro-leste do Estado de Minas Gerais. Revista Brasileira de Ciência do Solo, v. 34, n. 4, p. 1029-1039. 